\title{
ON $\mathbb{D}^{*}$-EXTENSION PROPERTY OF THE HARTOGS DOMAINS
}

\author{
Do Duc Thai and Pascal J. Thomas
}

\begin{abstract}
A complex analytic space is said to have the $\mathbb{D}^{*}$-extension property if and only if any holomorphic map from the punctured disk to the given space extends to a holomorphic map from the whole disk to the same space. A Hartogs domain $H$ over the base $X$ (a complex space) is a subset of $X \times \mathbb{C}$ where all the fibers over $X$ are disks centered at the origin, possibly of infinite radius. Denote by $\phi$ the function giving the logarithm of the reciprocal of the radius of the fibers, so that, when $X$ is pseudoconvex, $H$ is pseudoconvex if and only if $\phi$ is plurisubharmonic.

We prove that $H$ has the $\mathbb{D}^{*}$-extension property if and only if (i) $X$ itself has the $\mathbb{D}^{*}$-extension property, (ii) $\phi$ takes only finite values and (iii) $\phi$ is plurisubharmonic. This implies the existence of domains which have the $\mathbb{D}^{*}$-extension property without being (Kobayashi) hyperbolic, and simplifies and generalizes the authors' previous such example.
\end{abstract}

\section{Introduction}

The "big Picard" theorem states that any holomorphic map $f$ from the punctured unit disc $\mathbb{D}^{*}$ into the Riemann sphere $\mathbb{P}_{1}(\mathbb{C})$ which omits three points can be extended to a holomorphic map $f: \mathbb{D} \longrightarrow \mathbb{P}_{1}(\mathbb{C})$. Kwack $[\mathbf{K w}]$ extended this theorem to a higher dimensional context. If $f$ is a holomorphic map from $\mathbb{D}^{*}$ into a hyperbolic space $X$ such that, for a suitable sequence of points $z_{k} \in \mathbb{D}^{*}$ converging to the origin, $f\left(z_{k}\right)$ converges to a point $p_{0} \in X$, then $f$ extends to a holomorphic map from $\mathbb{D}$ into $X$.

2000 Mathematics Subject Classification. Primary 32H02, 32D20; Secondary 32H20, $32 \mathrm{~F} 30$.

Key words. Kobayashi hyperbolicity, removable singularities, Kontinuitätsatz, extension through pluripolar sets. 
The above-mentioned theorem of Kwack has strongly motivated the study of the extension problem of holomorphic maps through isolated singularities. At the same time, this result has suggested the study of the class of complex spaces having the following property.

Definition. Let $X$ be a complex space. We say that $X$ has the $\mathbb{D}^{*}$-extension property $\left(\mathbb{D}^{*}\right.$-EP) iff for any holomorphic map $f$ from $\mathbb{D}^{*}=$ $\{z \in \mathbb{C}: 0<|z|<1\}$ to $X$, there exists a map $\tilde{f} \in \operatorname{Hol}(\mathbb{D}, X)$ (where $\mathbb{D}=\{z \in \mathbb{C}:|z|<1\})$ such that $\left.\widetilde{f}\right|_{\mathbb{D}^{*}}=f$.

Much attention has been devoted to the $\mathbb{D}^{*}$-EP and various theorems have been obtained by Kwack $[\mathbf{K w}]$, Thai $[\mathbf{T h}]$, Thai and Thomas [Th-Tho], and others, see the monograph [Ko].

The first aim of this note is to prove the following

Theorem 1. Let $X_{\varphi}:=\left\{(z, w) \in X \times \mathbb{C}:|w|<e^{-\varphi(z)}\right\}$ where $\varphi: X \rightarrow$ $[-\infty,+\infty)$ is upper semi continuous.

Then $X_{\varphi}$ has the $\mathbb{D}^{*}-E P$ iff:

$X$ has the $\mathbb{D}^{*}-E P, \varphi \in P S H(X)$ and $\varphi(z)>-\infty, \forall z \in X$.

Notice that this result admits as a corollary, when $X=\mathbb{D}$ and $\varphi$ is not locally bounded, the existence of a domain in $\mathbb{C}^{2}$ which has the $D^{*}$-EP without being Kobayashi hyperbolic. We thus simplify the proof of that result given in [Th-Tho], and generalize somewhat the class of counter-examples available.

The proof of this result can be carried out by elementary means. However, use of more powerful theorems makes for shorter proofs and more general results. We denote by $\Lambda_{d}$ the Hausdorff measure in (real) dimension $d$.

Definition. We say that $X$ has the $n$-PEP (resp. $n$-PPEP, $(n, d)$-EP) iff for any closed set $A \subset \mathbb{D}^{n}$ which is polar (resp. pluripolar, resp. of locally finite $\Lambda_{d}$ measure), for any holomorphic map $f$ from $\mathbb{D}^{n} \backslash A$ to $X$, there exists a map $\tilde{f} \in \operatorname{Hol}\left(\mathbb{D}^{n}, X\right)$ (where $\mathbb{D}=\{z \in \mathbb{C}:|z|<1\}$ ) such that $\left.\widetilde{f}\right|_{\mathbb{D}^{n} \backslash A}=f$.

Re-using the notations of Theorem 1 , we have, for any $n \geq 1,2 n-2<$ $d<2 n-1$ :

Theorem 2. $X_{\varphi}$ has the $n-P E P$ (resp. n-PPEP, $(n, d)-E P$ ) iff:

$X$ has the $n$-PEP (resp. $n$-PPEP, $(n, d)-E P), \varphi \in P S H(X)$ and $\varphi(z)>-\infty, \forall z \in X$.

We would like to thank Ahmed Zeriahi and Nguyen Van Khue for their helpful suggestions. 


\section{Proof of Theorem 1}

\section{1) Sufficiency:}

Let $H \in \operatorname{Hol}\left(\mathbb{D}^{*}, X_{\varphi}\right), H=\left(h_{1}, h_{2}\right)$. Since $X$ has the $\mathbb{D}^{*}$-EP, $h_{1}$ extends to a map $\widetilde{h}_{1}, \widetilde{h}_{1}: \mathbb{D} \rightarrow X$. Let $z_{0}=\widetilde{h}_{1}(0)$. We need the following result on the local growth of (pluri-)subharmonic functions.

Theorem ([Ho1, Corollary 4.4.6, p. 98] or [Ho2, Corollary 4.2.10, p. 261]). If $\varphi \in P S H(\Omega) \backslash\{-\infty\}$, where $\Omega$ is a connected pseudoconvex open set, then $e^{-\varphi}$ is locally integrable in a dense open subset $G$ containing all points $z$ where $\varphi(z)>-\infty$.

In particular, since $\varphi\left(z_{0}\right)$ is finite, $e^{-2 \varphi} \in L_{\text {loc }}^{1}$ in a neighborhood of $z_{0}$. But then $h_{2} \in L_{\text {loc }}^{2}$, which implies that $h_{2}$ extends holomorphically across 0 , as can easily be deduced from the Laurent series expansion.

Finally, to see that we actually have $\log \left|\widetilde{h}_{2}(0)\right|<-\varphi\left(\widetilde{h}_{1}(0)\right)$, we apply the maximum principle to the subharmonic function $\log \left|\widetilde{h}_{2}\right|+\varphi\left(\widetilde{h}_{1}\right)$, as was done in [Th-Tho].

A direct proof of the extendability of $h_{2}$ may be given without recourse to Hörmander's result.

Since $\varphi$ is u.s.c, there exists $r_{0}>0, M \in \mathbb{R}$ such that $\varphi(z) \leq M$, $\forall z \in \bar{D}\left(z_{0}, r_{0}\right)$. Without loss of generality, suppose $M \leq 0$. Therefore we have

$$
\log \left|h_{2}(\xi)\right| \leq-\varphi\left(\widetilde{h}_{1}(\xi)\right)
$$

and since $\varphi \leq 0, \log _{+}\left|h_{2}(\xi)\right| \leq-\varphi_{1}(\xi)$, where $\varphi_{1}(\xi):=\varphi\left(\widetilde{h}_{1}(\xi)\right)$, therefore $\varphi_{1} \in \mathrm{SH}\left(\mathbb{D}, \mathbb{R}_{-}\right)$.

By the mean value inequality for subharmonic functions, (1) implies: $\forall r \in\left(0, r_{0}\right)$,

$$
\frac{1}{\pi r^{2}} \int_{D(0, r)} \log _{+}\left|h_{2}(\xi)\right| d \lambda_{2}(\xi) \leq-\varphi_{1}(0)<+\infty .
$$

We want to show that this implies that $h_{2}$ has a removable singularity at the origin.

Expand $h_{2}$ as a Laurent series

$$
h_{2}(\xi)=\sum_{n \in \mathbb{Z}} a_{n} \xi^{n} .
$$

Then for $r$ small enough,

$$
\left|\sum_{n>0} a_{n} \xi^{n}\right| \leq e
$$


SO

$$
\log _{+}\left|h_{0}(\xi)\right| \leq 1+\log _{+}\left|h_{2}(\xi)\right|
$$

where we set

$$
h_{0}(\xi):=\sum_{n \leq 0} a_{n} \xi^{n}
$$

and we are reduced to $\forall r>0$

$$
\frac{1}{\pi r^{2}} \int_{D(0, r)} \log _{+}\left|h_{0}(\xi)\right| d \lambda_{2}(\xi) \leq C<+\infty .
$$

Set $f(\xi):=h_{0}(1 / \xi)$. This is now an entire function. Under the change of variable $\psi=\frac{1}{\xi}$, we get

$$
\frac{1}{\pi r^{2}} \int_{D(0, r)} \log _{+}\left|h_{0}(\xi)\right| d \lambda_{2}(\xi)=\frac{1}{\pi r^{2}} \int_{\mathbb{C} \backslash \bar{D}(0,1 / r)} \log _{+}|f(\psi)| \frac{1}{|\psi|^{4}} d \lambda_{2}(\psi) .
$$

Now $\log _{+}|f| \in \mathrm{SH}(\mathbb{C})$, so

$$
m(\rho):=\int_{0}^{2 \pi} \log _{+}\left|f\left(\rho e^{i \theta}\right)\right| \frac{d \theta}{2 \pi}
$$

is an increasing function of $\rho$.

Passing to polar coodinates, we get that

$$
\begin{aligned}
C & \geq \frac{1}{\pi r^{2}} \int_{\frac{1}{r}}^{\infty}\left(\int_{0}^{2 \pi} \log _{+}\left|f\left(\rho e^{i \theta}\right)\right| d \theta\right) \frac{1}{\rho^{3}} d \rho \\
& =\frac{2}{r^{2}} \int_{\frac{1}{r}}^{\infty} \frac{m(\rho)}{\rho^{3}} d \rho \\
& \geq \frac{2}{r^{2}} m(1 / r) \int_{\frac{1}{r}}^{\infty} \frac{d \rho}{\rho^{3}}=m\left(\frac{1}{r}\right) .
\end{aligned}
$$

Therefore $m(\rho)$ is bounded as $\rho \rightarrow \infty$. But then, since $\log _{+}|f| \in \mathrm{SH}(\mathbb{C})$, it must be bounded above on $\mathbb{C}$, since by the Poisson formula

$$
\log _{+}\left|f\left(z_{0}\right)\right| \leq \int_{0}^{2 \pi} \frac{1-\left|\frac{z_{0}}{\rho}\right|^{2}}{\left|\frac{z_{0}}{\rho}-e^{i \theta}\right|^{2}} \log _{+}\left|f\left(\rho e^{i \theta}\right)\right| \frac{d \theta}{2 \pi} \leq \frac{1+\left|\frac{z_{0}}{\rho}\right|}{1-\left|\frac{z_{0}}{\rho}\right|} m(\rho) \leq 3 C,
$$

for $\rho \geq 2\left|z_{0}\right|$, so $f$ is constant by Liouville's theorem.

Therefore $h$ has a removable singularity at 0 . 


\section{2) Necessity:}

To prove that $X$ has the $\mathbb{D}^{*}$-EP, if $h \in \operatorname{Hol}\left(\mathbb{D}^{*}, X\right)$, then the map $H$ given by $H(\xi):=(h(\xi), 0)$ is holomorphic from $\mathbb{D}^{*}$ to $X_{\varphi}$ and must therefore admit an extension $\widetilde{H}$ such that (by continuity) $\widetilde{H}(\mathbb{D}) \subset X \times$ $\{0\} \subset X_{\varphi}$. Writing $\widetilde{H}=(\widetilde{h}, 0)$, we obtain the required extension of $h$.

If there exists $z_{0} \in X$ such that $\varphi\left(z_{0}\right)=-\infty$ then the complex line $\left\{\left(z_{0}, w\right): w \in \mathbb{C}\right\} \subset X_{\varphi}$, so $X_{\varphi}$ does not have the $\mathbb{D}^{*}$-EP (take $h(\xi)=\left(z_{0}, 1 / \xi\right)$, see $\left.[\mathbf{T h}]\right)$.

There remains to show that $\varphi \in P S H(X)$. We first do this for the case where $X$ is an open set in $\mathbb{C}^{n}$.

Lemma. Let $\Omega \subset \mathbb{C}^{n}$ be a domain with the $\mathbb{D}^{*}-E P$. Then $\Omega$ is pseudoconvex.

This lemma (which we alluded to in [Th-Tho]) is a consequence of a theorem of Shiffman [Si] (see also [So-Th]): if for any sequence $\left\{f_{n}\right\} \subset$ $\operatorname{Hol}(\mathbb{D}, X)$, convergence of $\left\{\left.f_{n}\right|_{\mathbb{D}^{*}}\right\}$ in $\operatorname{Hol}\left(\mathbb{D}^{*}, X\right)$ implies convergence of $\left\{f_{n}\right\}$ ("weak disk condition"), then $X$ has the Hartogs extension condition (which implies pseudoconvexity for open sets in $\mathbb{C}^{n}$ ).

But a domain in $\mathbb{C}^{n}$ with the $\mathbb{D}^{*}$-EP verifies the weak disk condition (simply extend the limit mapping and then apply the maximum principle on all coordinates).

However, there is a direct and elementary proof which avoids the use of Shiffman's theorem. For the reader's convenience, and since some colleagues of ours seemed to find it nice, we include it here.

Direct Proof of the Lemma: Let $\Phi$ be a holomorphic embedding of the closed unit bidisk $\overline{\mathbb{D}}^{2}$ into $\mathbb{C}^{n}$. Call Hartogs figure the image under $\Phi$ of the set $H_{0}:=\left\{\left|z_{1}\right| \leq 1, z_{2}=0\right\} \cup\left\{\left|z_{1}\right|=1,\left|z_{2}\right| \leq 1\right\}$. Recall that $\Omega$ is pseudoconvex if and only if for every Hartogs figure contained in $\Omega$, $\Phi\left(\mathbb{D}^{2}\right)$ is also contained in $\Omega[\mathbf{R a}]$.

Therefore, assuming $\Omega$ is not pseudoconvex, we obtain the following situation: there exists a holomorphic embedding $\Phi$ such that $\Omega_{1}:=$ $\Phi^{-1}(\Omega) \cap \mathbb{D}^{2}$ is open, $\mathbb{D}^{2} \backslash \Omega_{1} \neq \emptyset$, and $\overline{\mathbb{D}^{2} \backslash \Omega_{1}} \cap H_{0}=\emptyset$.

Let

$$
r_{2}:=\inf \left\{\left|z_{2}\right|:\left(z_{1}, z_{2}\right) \in \mathbb{D}^{2} \backslash \Omega_{1}\right\}
$$

our hypotheses mean that $0<r_{2}<1$, and they also imply that the set

$$
K:=\left(\mathbb{D}^{2} \backslash \Omega_{1}\right) \cap\left\{\left|z_{2}\right| \leq \frac{1+r_{2}}{2}\right\}
$$


is compact in $\mathbb{D}^{2}$, and therefore $r_{1}:=\max _{\left(z_{1}, z_{2}\right) \in K}\left|z_{1}\right|<1$. Now set

$$
\delta_{\varepsilon}(z):=\varepsilon\left|z_{1}\right|^{2}-\left|z_{2}\right|^{2} .
$$

There exists a point $z^{0}=\left(z_{1}^{0}, z_{2}^{0}\right)$ so that $\delta_{\varepsilon}\left(z^{0}\right)=\max _{K} \delta_{\varepsilon}$. For $\varepsilon$ small enough $\left(|\varepsilon| r_{1}^{2}-\left(\frac{1+r_{2}}{2}\right)^{2}<-r_{2}^{2}\right), z^{0} \in K \backslash\left\{\left|z_{2}\right|=\frac{1+r_{2}}{2}\right\}$, so there exists a neighborhood $V$ of $z^{0}$ such that $V \cap \Omega_{1}=V \backslash K$. It is now enough to find an analytic disk $f$ with center $f(0) \in K$ and $f(D(0, r) \backslash\{0\}) \subset V \backslash K$, for $r>0$ small enough. We may in fact pick an affine disk, namely

$$
f(\xi):=\left(z_{1}^{0}+\bar{z}_{2}^{0} \xi, z_{2}^{0}+\varepsilon \bar{z}_{1}^{0} \xi\right) .
$$

Observe that $f(\mathbb{C})$ is a line tangent to the level hypersurface of $\delta_{\varepsilon}$ corresponding to the value $\delta_{\varepsilon}\left(z^{0}\right)$, and in fact an elementary calculation shows that

$$
\delta_{\varepsilon}(f(\xi))=\delta_{\varepsilon}\left(z^{0}\right)+\varepsilon|\xi|^{2}\left(\left|z_{2}^{0}\right|^{2}-\varepsilon\left|z_{1}^{0}\right|^{2}\right)>\delta_{\varepsilon}\left(z^{0}\right)
$$

for $\varepsilon>0$ and small enough $\left(z_{1}^{0}\right.$ and $z_{2}^{0}$ do depend on $\varepsilon$, but we have the condition as soon as $\left.r_{2}^{2}-\varepsilon r_{1}^{2}>0\right)$, which completes the proof of the lemma.

We may remark that setting $f_{t}(\xi):=f(\xi)+t \nabla \delta_{\varepsilon}\left(z^{0}\right)$, the map $\Phi$ 。 $f$ gives a disk violating the $\mathbb{D} *$-EP for the $\Omega$ which we had assumed non-pseudoconvex, and the maps $\Phi \circ f_{t}$ provide a refined failure of the Kontinuitätsatz for $\Omega$ (contact with the boundary occurs at one point exactly).

Together with the above lemma, the following will complete the proof of necessity.

Claim. If $X_{\varphi}$ has the $\mathbb{D}^{*}-E P$, and $\varphi \notin P S H(X)$ then there exists $\Omega \subset$ $\mathbb{C}^{2}$, having the $\mathbb{D}^{*}-E P$ and $\Omega$ not pseudoconvex.

We need the following characterization. Denote by $\mathcal{H} a(\mathbb{D})$ the space of harmonic functions on the disk.

Fact. $\varphi \in \operatorname{PSH}(X)$ iff $\forall f \in \operatorname{Hol}(\overline{\mathbb{D}}, X), \forall u \in \mathcal{H} a(\mathbb{D}) \cap \mathcal{C}^{0}(\overline{\mathbb{D}})$, such that $\varphi \circ f\left(e^{i \theta}\right) \leq u\left(e^{i \theta}\right) \forall \theta \in \mathbb{R}$, then $\varphi \circ f(0) \leq u(0)$.

This follows immediately from the theorem of Fornaess and Narasimhan $[\mathbf{F o}-\mathbf{N a}]$ which characterizes plurisubharmonic functions on complex spaces as those whose pullback under any analytic disk is subharmonic, and the characterization of subharmonicity by the mean value inequality (see e.g. [Ho1, Theorem 1.6.3, p. 16]).

Now suppose $\varphi \notin \operatorname{PSH}(X)$. Then $\exists f \in \operatorname{Hol}(\overline{\mathbb{D}}, X), u \in \mathcal{H} a(\mathbb{D}) \cap \mathcal{C}^{0}(\overline{\mathbb{D}})$, such that $\varphi(f(0))>u(0), \varphi\left(f\left(e^{i \theta}\right)\right) \leq u\left(e^{i \theta}\right), \forall \theta \in \mathbb{R}$. 
Let

$$
\begin{aligned}
\Omega:=\{(z, w) \in \mathbb{D} \times \mathbb{C}: & \left.(f(z), w) \in X_{\varphi}\right\} \\
& =\left\{(z, w) \in \mathbb{D} \times \mathbb{C}:|w|<e^{\varphi \circ f(z)}\right\}=: \mathbb{D}_{\varphi \circ f} .
\end{aligned}
$$

Since $\varphi \circ f$ is not subharmonic, the classical result about Hartogs domains implies that $\Omega$ is not pseudoconvex. The following claim then completes our proof.

Claim. $\Omega$ has the $\mathbb{D}^{*}-E P$.

Proof of the Claim: Let $h \in \operatorname{Hol}\left(\mathbb{D}^{*}, \Omega\right), h(\xi)=\left(h_{1}(\xi), h_{2}(\xi)\right)$.

Now $h_{1}(\xi) \in \mathbb{D}$ for all $\xi$, so $h_{1}(\xi)$ extends to $\widetilde{h}_{1} \in \operatorname{Hol}(\mathbb{D}, \mathbb{D})$. The map $\xi \mapsto\left(f \circ h_{1}(\xi), h_{2}(\xi)\right)$ is holomorphic from $\mathbb{D}^{*}$ to $X_{\varphi}$ by construction, so it extends to $F \in \operatorname{Hol}\left(\mathbb{D}, X_{\varphi}\right)$.

Let $F(\xi)=\left(F_{1}(\xi), F_{2}(\xi)\right)$.

$F_{2}$ provides an extension of $h_{2}$. It remains to see that $\widetilde{h}=\left(\widetilde{h}_{1}, F_{2}\right) \in$ $\operatorname{Hol}(\mathbb{D}, \Omega)$, that is, that $\left|f\left(\widetilde{h}_{1}(0)\right)\right|<e^{-\varphi\left(F_{2}(0)\right)}$.

Since $\left|f\left(\widetilde{h}_{1}(0)\right)\right|=\lim _{\xi \rightarrow 0}\left|f\left(h_{1}(\xi)\right)\right|=\lim _{\xi \rightarrow 0}\left|F_{1}(\xi)\right|=\left|F_{1}(0)\right|<$ $e^{-\varphi\left(F_{2}(0)\right)}$ because $F \in \operatorname{Hol}\left(\mathbb{D}, X_{\varphi}\right)$, we are done.

\section{Proof of Theorem 2}

The direct implication proceeds as in the previous section, noticing that each of the extension properties we have defined implies the $\mathbb{D}^{*}$-EP. (In the case where $n \geq 2$, given a map $f \in \operatorname{Hol}\left(\mathbb{D}^{*}, X\right)$, simply consider the map $F \in \operatorname{Hol}\left(\mathbb{D}^{n} \backslash\left\{z_{1}=0\right\}, X\right)$ given by $F\left(z_{1}, \ldots, z_{n}\right):=f\left(z_{1}\right)$.)

To prove the converse implication, recall the following result of extension.

Theorem ([Ha-Po, Theorem 1, (d)]). Suppose $A$ is a closed subset of an open set $\Omega \subset \mathbb{C}^{n}$ and that $f \in \operatorname{Hol}(\Omega \backslash A)$. Let $2 \leq p<\infty$ and $p^{\prime}$ be the conjugate exponent $\left(\frac{1}{p}+\frac{1}{p^{\prime}}=1\right)$. If $f \in L_{\mathrm{loc}}^{p}(\Omega)$ and $\Lambda_{2 n-p^{\prime}}(A)$ is locally finite, then $f \in \operatorname{Hol}(\Omega)$.

Now given $d$ as in the theorem, set $p^{\prime}:=2 n-d$ and $p$ its conjugate exponent. Given a map $h=\left(h_{1}, h_{2}\right) \in \operatorname{Hol}\left(\mathbb{D}^{n} \backslash A, X_{\varphi}\right), h_{1}$ extends to $\widetilde{h}_{1} \in \operatorname{Hol}\left(\mathbb{D}^{n}, X\right)$ by the extension property for $X$ which is included in the hypothesis, and $p \varphi \circ \widetilde{h}_{1}$ is a finite-valued plurisubharmonic function, so locally integrable, therefore $\left|h_{2}\right| \leq e^{-\varphi \circ \widetilde{h}_{1}}$ verifies all the hypotheses of the Harvey-Polking theorem. 
If $A$ is polar, it is a classical fact that $\Lambda_{d}(A)=0$ for any $d>2 n-2$ $[\mathbf{L a}]$ and the same is true for pluri-polar sets $[\mathbf{K l}]$.

\section{References}

[Fo-Na] J. E. Forness and R. NARASImHan, The Levi problem on complex spaces with singularities, Math. Ann. 248(1) (1980), $47-72$.

[Ha-Po] R. Harvey and J. Polking, Extending analytic objects, Comm. Pure Appl. Math. 28(6) (1975), 701-727.

[Ho1] L. HöRmANDER, "An introduction to complex analysis in several variables", third ed., North-Holland Mathematical Library 7, North-Holland Publishing Co., Amsterdam, 1990.

[Ho2] L. Hörmander, "Notions of convexity", Progress in Mathematics 127, Birkhäuser Boston Inc., Boston, MA, 1994.

[Kl] M. KLIMEK, "Pluripotential theory", Oxford Science Publications London Mathematical Society Monographs. New Series 6, The Clarendon Press Oxford University Press, New York, 1991.

[Ko] S. Kobayashi, "Hyperbolic complex spaces", Grundlehren der Mathematischen Wissenschaften 318, Springer-Verlag, Berlin, 1998.

[Kw] M. H. KWACK, Generalization of the big Picard theorem, Ann. of Math. (2) 90 (1969), 9-22.

[La] N. S. LANDKOF, "Foundations of modern potential theory", Die Grundlehren der mathematischen Wissenschaften 180, Springer-Verlag, New York, 1972.

[Ra] R. M. RANGE, "Holomorphic functions and integral representations in several complex variables", Graduate Texts in Mathematics 108, Springer-Verlag, New York, 1986.

[Sh] B. V. Shabat, "Introduction to complex analysis. Part II", Functions of several variables, Translations of Mathematical Monographs 110, American Mathematical Society, Providence, RI, 1992.

[Si] B. Shiffman, Extension of holomorphic maps into Hermitian manifolds, Math. Ann. 194 (1971), 249-258.

[So-Th] N. T. Son AND D. D. ThaI, Extensions of holomorphic maps through hypersurfaces and relation to the Hartogs extension in infinite dimension, Proc. Amer. Math. Soc. (to appear).

[Th] D. D. ThAI, On the $\mathbb{D}^{*}$-extension and the Hartogs extension, Ann. Scuola Norm. Sup. Pisa Cl. Sci. (4) 18(1) (1991), 13-38. 
[Th-Tho] D. D. Thai And P. J. Thomas, $\mathbb{D}^{*}$-extension property without hyperbolicity, Indiana Univ. Math. J. 47(3) (1998), 1125-1130.

Do Duc Thai:

Department of Mathematics

Institute of Pedagogy no 1

Cau Giay

Hanoi

Vietnam

E-mail address: ddthai@netnam.org

Pascal J. Thomas:

Laboratoire de Mathématiques Emile Picard

CNRS UMR 5580

Université Paul Sabatier

118 route de Narbonne

31062 Toulouse Cedex

France

E-mail address: pthomas@cict.fr

Rebut el 9 d'octubre de 2000. 